\title{
Discours
}

Revue de linguistique, psycholinguistique et informatique. A journal of linguistics, psycholinguistics and computational linguistics

$6 \mid 2010$

Le fonctionnement en discours des énoncés averbaux autonomes

\section{Focalisation averbale $v s$ focalisation verbale en français parlé}

Le cas des constructions binaires

\section{Noalig Tanguy}

\section{(2) OpenEdition}

\section{Journals}

Édition électronique

URL : http://journals.openedition.org/discours/7726

DOI : $10.4000 /$ discours. 7726

ISSN : 1963-1723

Éditeur :

Laboratoire LATTICE, Presses universitaires de Caen

\section{Référence électronique}

Noalig Tanguy, «Focalisation averbale vs focalisation verbale en français parlé », Discours [En ligne],

6 | 2010, mis en ligne le 29 septembre 2010, consulté le 20 avril 2019. URL : http://

journals.openedition.org/discours/7726 ; DOI : 10.4000/discours.7726

Ce document a été généré automatiquement le 20 avril 2019

\section{c) $($ ) $(9)$}

Discours est mis à disposition selon les termes de la licence Creative Commons Attribution - Pas d'Utilisation Commerciale - Pas de Modification 4.0 International. 


\title{
Focalisation averbale vs focalisation verbale en français parlé
}

\author{
Le cas des constructions binaires
}

Noalig Tanguy

1 De nombreux travaux sur la prédication averbale en français (Le Goffic, 1993; Lefeuvre, 1999 et 2007) ont vu le jour ces dernières années et l'existence de la phrase sans verbe n'est plus à prouver. Simultanément, diverses études sur de larges corpus de français parlé (notamment Blanche-Benveniste, 1990, 1997 et 2002a ; Deulofeu, 2003 ; Sabio 1995, 2006a, 2006b et 2007; Morel et Danon-Boileau, 1998; Berrendonner, 1990; etc.) ont permis de mettre en lumière des structures bien particulières trop souvent mal définies, comme par exemple des énoncés du type :

[1] le déroulement de la guerre est-ce qu'il y avait des des informations qui circulaient (CTFP : 29) ${ }^{1}$

[2] il y a mon frère tu vois / sa moto / le guidon / eh ben tout cassé quoi \_ce truc ---_ (Deulofeu, 2003)

[3] c'était une croisière // sur un un bateau un joli bateau // qui est arrêté maintenant $/ /^{2}$ (CRFP, DELIC, 2005) $)^{3}$

[4] Une fortune ça m'a coûté ! (Blanche-Benveniste, 2002a)

qui présentent des structures non conventionnelles : un élément disloqué non régi en [1], également appelé «nominativus pendens », comme premier fragment n'entretenant aucun rapport de dépendance syntaxique avec le reste de la phrase (Berrendonner, 2003), une succession de segments averbaux en [2], une structure phrastique fragmentée en plusieurs unités prosodiques en [3] et une antéposition d'un complément régi en [4].

2 Nous nous proposons dans cet article de rapprocher deux types de structures couramment employées en français parlé qui présentent toutes deux la particularité d'être énoncées en deux temps. Cependant la première, du type de [4], est construite autour d'un verbe, tandis que la seconde construction est averbale :

[5] à la caisse ils se pèsent (Sabio, 1995)

[6] très bien ta vie (CTFP : 32) 
Après avoir décrit leur fonctionnement à partir du modèle qui est le nôtre, nous verrons que ces deux types de réalisation résultent d'une même opération de focalisation, opération visant à placer en tête de phrase un argument à vocation rhématique, et que les deux structures syntaxiques sont en réalité deux variantes combinatoires d'un même procédé, construites sur le même moule intonosyntaxique avec des effets informationnels similaires.

\section{Principes théoriques}

\subsection{La phrase sans verbe}

4 Il est admis aujourd'hui qu'un énoncé sans verbe peut tout à fait constituer le centre prédicatif d'une phrase, si l'on entend comme définition de celle-ci une prédication en acte assortie d'une modalité énonciative (Le Goffic, 2008). La phrase est donc une unité à la fois énonciative et syntaxique. Nous entendons par prédication l'opération sémanticosyntaxique de construction d'un énoncé, la mise en relation d'un sujet et d'un prédicat. Nous distinguons cinq modes de prédication: d'existence, d'attribution, de localisation, d'activité et de cognitivité. Les phrases verbales peuvent réaliser tous ces énoncés. Les phrases averbales sont limitées aux trois premiers modes de prédication cités. Le rôle de prédicat peut ainsi être tenu par un constituant averbal: un syntagme nominal (substantival ou pronominal), un groupe adjectival (ou participial), un groupe adverbial ou un syntagme prépositionnel.

5 Nous rappelons très brièvement que trois cas de figures sont possibles pour la phrase averbale.

6 (i) Une construction à deux termes comporte les éléments « sujet » et " prédicat » selon les deux ordres possibles.

[7a] très bien ce que tu as fait (CTFP : 32)

$[7 \mathrm{~b}]$ ce que tu as fait très bien

7 La notion de « sujet » est cependant à modérer pour les prédications averbales puisqu'il s'agit en réalité d'un élément suppressible. Il n'est pas indispensable, comme l'est le prédicat. Cela revient à notre deuxième configuration possible.

8 (ii) Dans les structures dites «à un terme" (Lefeuvre, 1999: 41), seul le prédicat est formulé. Le sujet y est implicite et son référent est présent dans le contexte situationnel ou linguistique.

[8] très beau (CTFP : 32)

[9] d'accord (CTFP : 11)

9 (iii) Enfin, nous avons une structure sans sujet dite « existentielle ».

[10] en face de moi un tapis roulant euh qui menait directement euh à des jolies demoiselles (CTFP : 30 )

Dans ce cas, le noyau prédicatif marque une existence. Il est très souvent précédé de circonstants cadratifs et extra-prédicatifs comme ici avec le groupe prépositionnel « en face de moi ». La construction suit alors une structure binaire en « thème-rhème ». 


\subsection{Structuration du français parlé}

10 Parallèlement aux avancées des études sur les énoncés averbaux, les recherches sur le français parlé ont offert de nouveaux outils pour traiter des unités de l'oral, verbales comme averbales. Toutes dégagent des structures tripartites :

11 (i) «préfixe + noyau + suffixe / postfixe » (Blanche-Benveniste, 1990 et 1997) ou «prénoyau + noyau + postnoyau » (Deulofeu, 2003)

12 (ii) « préambule + thème + postrhème » (Morel et Danon-Boileau, 1998)

13 (iii) « support + apport + report » (Perrot, 1978, 1994 et 1995), etc.

définies selon des critères divers: (i) approche pronominale et relations macrosyntaxiques réalisées par des marques intonatives et/ou des regroupements sémantiques pour les approches aixoises, (ii) unités d'analyse intonatives et discursives dans le modèle de Morel et Danon-Boileau, (iii) valeurs prosodiques et informationnelles distinguant information subordonnée, principale et retardée chez Perrot.

14 Cette structuration triple semble pouvoir tout à fait s'appliquer à la définition de la phrase telle que nous l'entendons, à savoir un prédicat, lié ou non à un sujet, et au(x)quel (s) se rattachent des éléments périphériques, avant ou après, plus ou moins extérieurs au prédicat. Nous reprendrons ainsi pour notre étude la terminologie de Blanche-Benveniste appliquée à un modèle syntaxique autour de la notion de phrase. Nous ne retiendrons cependant du modèle aixois que la terminologie. Nous travaillerons à partir du modèle suivant :

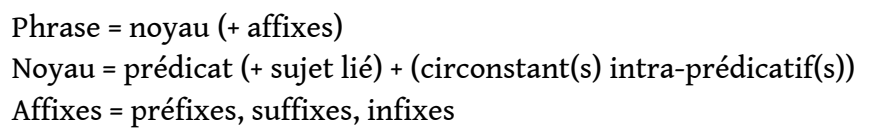

Fig. 1 : modèle théorique d'analyse syntaxique du français parlé

Ainsi, une phrase comporte nécessairement un noyau et éventuellement des affixes. Ce noyau réalise un prédicat et très souvent son sujet en structure liée. Il comprend également les circonstants internes au prédicat (dits « intra-prédicatifs »). Il peut reposer sur un prédicat verbal (phrase verbale) ou sur un prédicat averbal (phrase averbale). Les affixes, selon leur place, peuvent être préfixes, suffixes ou infixes.

Dans les structures averbales à deux termes (en «sujet-prédicat» ou en "prédicatsujet »), le sujet peut parfois être analysé comme un affixe lorsqu'il apparait détaché et suppressible. C'est le cas de [11] par exemple :

$$
\text { [11] très bien [noyau] ta vie [suffixe] (CTFP : 32) }
$$

Nous nuancerons plus loin dans notre démonstration la notion de sujet pour ce type d'énoncé.

\section{Zoom sur les structures binaires}

Nous souhaitons à présent confronter deux constructions en apparence éloignées mais qui présentent en réalité un fonctionnement relativement similaire. Nous décrirons ainsi successivement : 
(i) les phrases averbales à deux termes (à sujet explicite) qui s'articulent selon l'ordre le plus fréquent pour les structures sans verbe et binaires, "prédicat-sujet ", avec sujet détaché en fin de phrase et donc analysable en terme de suffixe, du type :

[12] À paris le vernissage ? (oral) position de noyau et en tête de phrase, comme par exemple :

[13] à Marseille il habitait (Sabio, 2007)

construction typique de l'oral, analysée selon les approches comme des "postfixes » (Sabio, 1995 ; Blanche-Benveniste, 1997) ou des « clauses disloquées » (Béguelin, 2003).

Ces deux types d'énoncés présentent des points communs même s'ils se distinguent à plusieurs niveaux. Il s'agit tout d'abord de formes phrastiques non canoniques et non liées résultant d'une opération focalisante. Cependant, l'une est averbale alors que l'autre se construit autour d'un verbe. De plus, la première projette le prédicat en tête de phrase tandis que la seconde relègue la partie verbale, donc la tête prédicative, en fin d'énoncé.

\subsection{Les phrases averbales en prédicat-sujet}

21 Les phrases averbales à deux termes, à l'écrit comme à l'oral, connaissent deux ordres possibles : l'ordre « sujet-prédicat » qui s'apparente plus à l'ordre phrastique canonique et l'ordre "prédicat-sujet " plus typique de ces constructions. Nous nous intéresserons à ce dernier cas même si pour le français parlé, les structures averbales privilégiées sont les constructions à un terme (à sujet implicite ou les existentielles sans sujet).

Dans ce type de configuration, la phrase est dépourvue de verbe et c'est un constituant averbal qui porte la prédication.

En [6]:

[6] très bien ta vie (CTFP : 32)

le prédicat est relié au sujet par la modalité énonciative, ici assertive. L'énonciateur émet un jugement. Il asserte, affirme que le prédicat « très bien » convient au sujet « ta vie ».

Le prédicat averbal relève de différentes catégories syntaxiques: un groupe adjectival comme [14], un groupe substantival en [15], un groupe adverbial pour [7] ou encore un groupe prépositionnel comme en [16] :

[14] pas frais mon rouget (Blanche-Benveniste, 1990)

[15] des conneries ton truc (CTFP : 36)

[7] très bien ce que tu as fait (CTFP : 32)

[16] en sa possession hein le chèque (CTFP : 26)

Le sujet, quant à lui, subit de fortes contraintes sur sa détermination. Interprété comme déjà connu des interactants, le sujet est toujours défini et spécifique, caractéristique typique de ces structures disloquées. Ce sont ces deux aspects qui présupposent l'existence déjà connue du référent du sujet.

Les groupes substantivaux sujets sont introduits par différents déterminants définis : un article défini en [16] et un déterminant possessif pour [14] et [15]. Le groupe substantival « ce que tu as fait » en [7] est construit à partir du démonstratif « ce ». 
Le sujet qui d'ordinaire représente le point de départ de la construction sémantique est dans ce type de phrase énoncé " après coup ». Il est ainsi fortement mis en exergue. La thématisation est alors dite "maximale » (Lefeuvre, 1999: 185) : la suppression du sujet est possible, on perçoit une forte rupture intonative entre les deux éléments, une inversion des termes "prédicat» et «sujet» est facilement réalisable, etc. Cependant, cette position en fin de phrase s'apparente aux dislocations à droite du type :

[7c] c'est très bien ce que tu as fait

[14b] il est pas frais mon rouget

Cela rejoint ici le point de vue de Le Goffic (1993: 510) pour qui ce type d'élément « fait plutôt penser à un thème postposé qu'à un sujet au sens strict ». C'est davantage la dislocation d'un élément sujet implicite :

$$
\text { [14c] [il n'est] pas frais mon rouget }
$$

Nous parlerons donc avec prudence de « sujet » pour les constructions averbales. En effet, il ne s'agit pas de sujet au sens strict du terme. L'exemple [26c] que nous étudierons plus loin:

[26c] Intéressant, la Patagonie. (Blanche-Benveniste, 1990 : 141)

met en évidence cette objection. En effet, la définition du sujet comme terme donnant ses marques de genre et de personne au prédicat ne peut s'y appliquer. Syntaxiquement, le terme détaché ne peut être sujet comme le montre l'accord. Il a toutes les propriétés d'une dislocation à droite. Cependant, sémantiquement, il constitue le support de l'information de l'énoncé.

La structure averbale serait alors le résultat d'un effacement du verbe «être » et d'un sujet pronominal clitique, ou du moins, la structure focalisante serait possible sans verbe puisqu'une attribution peut être réalisée par un verbe attributif, mais elle peut l'être également par un terme averbal, comme ici le groupe adjectival "pas frais». Nous rappelons d'ailleurs à ce propos que les phrases sans verbe se construisent à partir du modèle des énoncés avec "être ". Comme eux, la prédication averbale marque une attribution, une localisation ou une existence. Nous verrons plus loin que cette hypothèse se confirmera avec le parallèle effectué entre les phrases averbales binaires et les constructions verbales à complément extraposé.

\subsection{Les compléments averbaux antéposés en position de noyau}

Une construction particulière en français parlé consiste à employer en tête de phrase un complément essentiel sous la dépendance syntaxique d'un verbe recteur. Elle réalise en effet en tête de phase un complément détaché et antéposé au verbe, mais obligatoire. Ainsi en [17] :

[17] un petit mouton il va m'acheter (Blanche-Benveniste, 1990 : 141)

la valence du verbe "acheter " appelle nécessairement la réalisation d'un complément essentiel direct. Cette construction peut surprendre puisque les compléments du verbe sont habituellement «non mobile[s] à l'intérieur du groupe verbal » (Riegel et al., 1994 : 222). 
Peuvent, dans ce type de structure, être détachés en début d'énoncé : des constituants adjectivaux, substantivaux, prépositionnels, en fonction d'attribut, de complément direct, indirect, etc. :

[18] aux vacances je renonce (Béguelin, 2003)

[19] deux cigarettes j'ai fumé (Sabio, 2007)

[20] somptueux il était (Sabio, 2007)

Il convient cependant de distinguer deux types d'antéposition de compléments essentiels verbaux. Nous reprendrons ici l'opposition formulée par Sabio (1995 et 2006b) qui dissocie les antépositions sous forme de préfixes, à valeur topicalisante, et les antépositions sous forme de noyaux, à valeur focalisante. Cette distinction est illustrée par l'opposition :

[21a] les F3 elle supporterait pas (Sabio, 2006b)

[22a] que de la tendresse tu m'inspires (Sabio, 2006b)

En [21a], les modalités touchent le verbe recteur. Les manipulations syntaxiques suivantes sont ainsi possibles :

[21b] les F3 elle supporterait vraiment pas

[21c] les F3 elle supporterait?

Pour [22a], les marques modales affectent l'élément antéposé :

[22b] rien que de la tendresse tu m'inspires

[22c] pas que de la tendresse tu m'inspires

Le procédé que nous étudions du type de [22a] a reçu, nous l'avons déjà mentionné, diverses appellations et interprétations: "clause disloquée » (Béguelin, 2003) ou «noyau» pour le premier élément, «postfixe »" (Blanche-Benveniste, 1997) ou « postnoyau» (Sabio, 2007) pour le second, position spécifique des éléments dits « afterthought ».

D'après la définition de la phrase que nous avons adoptée, c'est d'ordinaire le verbe, base du prédicat, qui constitue le noyau de toute phrase. Nous aurions ainsi pu dans un premier temps analyser ce type de structure en "préfixe-noyau " puisque d'un point de vue syntaxique, c'est le noyau qui renferme l'élément verbal, prédicatif, et que tout élément formulé avant est interprété comme un préfixe. Nous pensons au contraire qu'ici, c'est le syntagme averbal qui porte la véritable prédication. La partie verbale, quant à elle, est recatégorisée en position de suffixe. Le groupe prépositionnel nominal "à la caisse " fonctionne d'ailleurs sans problème comme prédicat dans une structure averbale du type de [5b] :

[5a] à la caisse ils se pèsent (Sabio, 1995 : 114)

[5b] à la caisse la pesée

Le contenu lexical, ici faible du verbe "peser ", dans cette construction où l'information essentielle est focalisée dans "à la caisse », permet cette analyse. De plus, cette partie verbale n'est pas strictement nécessaire à l'inverse de son complément. Le complément antéposé peut très bien fonctionner seul comme réponse à une question par exemple :

[5c] Où les fruits se pèsent-ils ? À la caisse. 
Cette construction s'oppose ainsi radicalement aux dislocations à gauche avec reprise par un pronom anaphorique dans le noyau, c'est-à-dire avec un double marquage (BlascoDulbecco, 1999), et pour lesquelles le complément thématisé en tête de phrase, en prolepse, est facultatif.

Nous reconnaissons tout de même le caractère délicat d'une telle interprétation offrant la qualité de prédicat à un élément rectionnel et reléguant la partie verbale à un statut périphérique.

\section{Deux variantes combinatoires d'une même opération}

\subsection{Aspects prosodiques}

Les éléments extraposés ont fait l'objet de nombreuses analyses et travaux au niveau prosodique. Un tel type de focalisation s'accompagne en effet de propriétés intonatives spécifiques. Pour Morel et Danon-Boileau (1998: 64) par exemple, cela prend la forme d'une montée très rapide de F0 et d'une forte augmentation de l'intensité à la finale du constituant focalisé appelé « focus », suivies d'une chute mélodique sur le " postfocus ».

De son côté, Blanche-Benveniste (1990 : 142) fait remarquer, tout comme Sabio (2006b), qu'un élément régi et antéposé peut connaître deux types d'intonation. Il connaît une intonation de préfixe dans des énoncés du type de :

[23] du nom / je ne me rappelle plus (Blanche-Benveniste, 1990)

où le groupe prépositionnel « du nom » est hors de la portée de la modalité du verbe, peut être supprimé sans altérer le sens de l'énoncé, et joue le rôle classique de «thème ». À l'inverse, l'élément focalisé est énoncé avec une intonation de noyau lorsque l'élément de rection est porteur des modalités de la phrase :

[4] Une fortune, ça m'a coûté ! (Blanche-Benveniste, 2002a)

41 Dans ce cas, le complément régi est rhématique. Il est actualisé par une mélodie descendante. Plus précisément, il se termine par un ton bas à valeur conclusive (Sabio, 2006b), typique des fins d'énoncés assertifs. Le reste de la construction est énoncé avec une intonation postfinale d'appendice (Blanche-Benveniste, 2002a; Mertens, 2006). Comme pour les structures clivées, le reste du prédicat (donc ici la partie verbale) non focalisé est énoncé avec une intonation basse et non modulée. Il est produit sans aucun relief intonatif et se rapproche ainsi des parenthèses (Sabio, 2006b).

C'est exactement le même contour prosodique que l'on retrouve dans nos énoncés averbaux, pour mémoire :

[7] très bien ce que tu as fait (CTFP : 32)

[24] 1 rue Pascal Marie Agasse ça s'appelle $(\mathrm{PFC})^{5}$ 


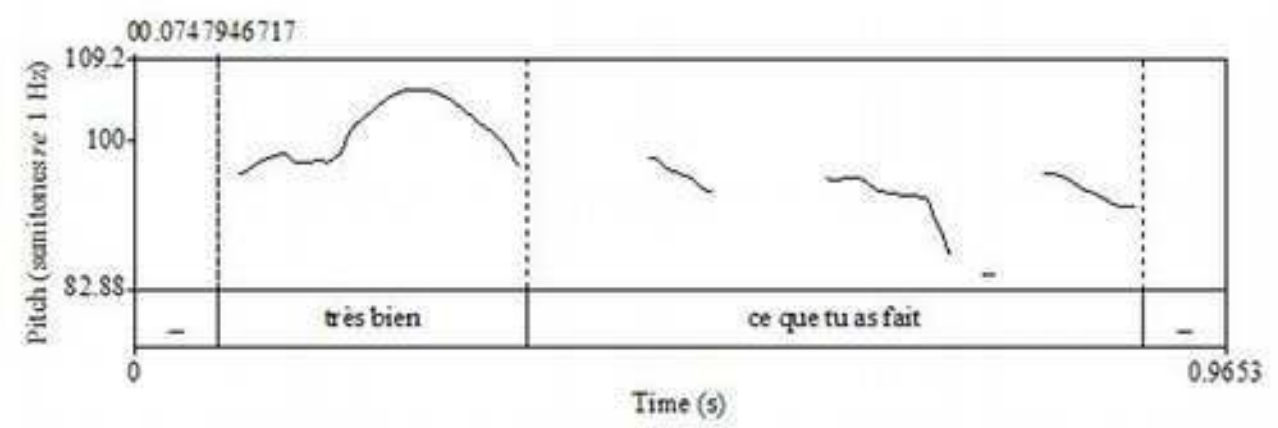

Fig. 2 : tracé mélodique de l'énoncé « très bien ce que tu as fait » (corpus CTFP : 32)

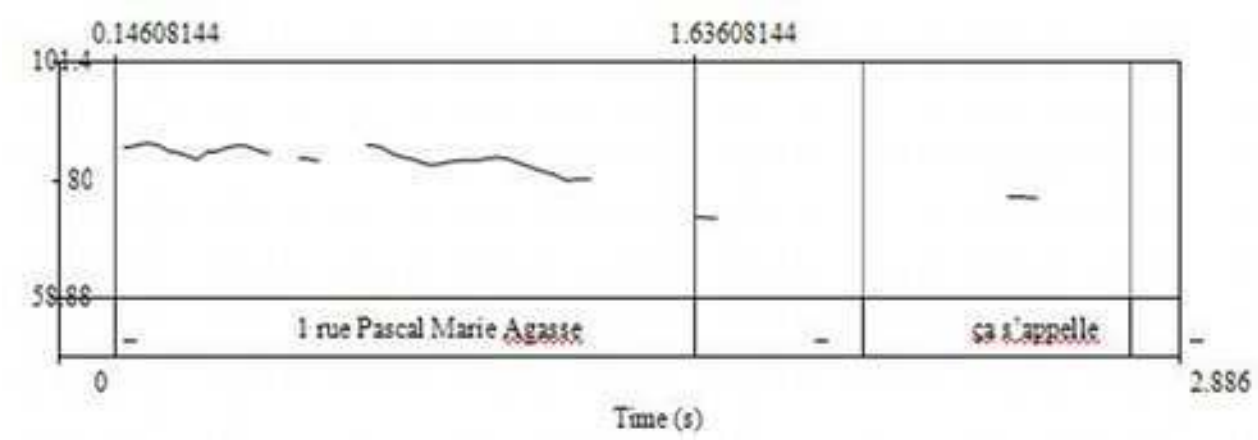

Fig. 3 : tracé mélodique de l'énoncé « 1 rue Pascal Marie Agasse ça s'appelle » (corpus PFC)

Les tracés prosodiques des énoncés [7] et [24] qui figurent ci-dessus ont été obtenus à l'aide du logiciel Praat (Boersma et Weenick, 2009). Ils montrent que les constructions à l'étude partagent les mêmes propriétés intonatives. Les deux sont prononcées en deux groupes intonatifs. Le premier est porteur d'une intonation modale qui le rend autonome. Le second est prononcé comme un appendice. La forme de l'intonation modale du premier terme (élément focalisé) peut varier. Pour preuve, dans l'exemple [7], le contour de la première construction est en cloche ${ }^{6}$, alors que dans l'énoncé [24], il est descendant. Quant au second segment, il est prononcé sur une plage plus basse que le contour qui précède et duquel il constitue en quelque sorte le prolongement. Dans l'exemple [24], la F0 n'est presque pas visible, car proche du chuchotement. Enfin, nous remarquons qu'une pause peut séparer les deux constructions averbales (exemple [24]). Cela n'est cependant pas obligatoire (pause dans le second mais pas dans le premier).

\subsection{Processus d'extraction}

Ces deux modèles de constructions ressortent tous deux d'un même processus de focalisation interférant sur l'organisation phrastique. Le modèle de la phrase canonique, dans lequel le sujet est entendu en toute logique comme un topique à propos duquel le prédicat formule un commentaire, se voit ainsi bousculé. Ce moule normatif français en « sujet + prédicat verbal », en "S-V-O », ou encore en « [SN V (SX)]» (Lambrecht, 2008) est d'ailleurs assez rarement employé en français parlé qui use davantage de structures particulières à l'oral spontané tendant à segmenter le discours plus que l'écrit.

La prédication averbale en deux termes que nous étudions emploie un prédicat en tête de phrase. Le sujet est exprimé après coup, en fin d'énoncé. Le locuteur accentue ainsi avec 
insistance l'énonciation de l'élément prédicatif jugé essentiel sur le plan informatif. Cette mise en relief s'oppose à la construction canonique, liée, avec « être ", et analysable en un seul élément «noyau» :

[7a] très bien ce que tu as fait (CTFP : 32)

$[7 \mathrm{~d}]$ ce que tu as fait est très bien

Pour reprendre les termes de Lambrecht (1994, 2004 et 2008), nos constructions permettent de marquer comme [foc+] un élément qui serait autrement interprété comme [foc-].

C'est le même principe pour l'antéposition d'un complément essentiel. Lambrecht parle de «focus sur argument ». La construction binaire permet de coder un argument focal dans une proposition présupposée.

Nous sommes ici très proches du principe des constructions clivées (Lambrecht, 2004) qui permet également de mettre en valeur tel ou tel élément selon son degré d'importance :

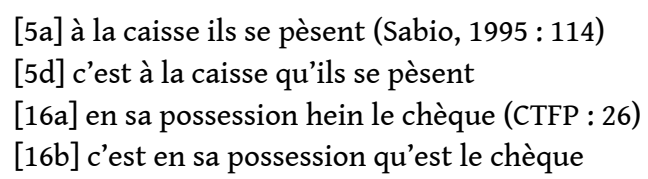

L'acte de la focalisation, tout comme celui du clivage, repose sur le principe qu'il ne peut porter que sur un élément rhématique (Morel et Danon-Boileau, 1998 : 64). Nos structures accordent une place de premier ordre à des constituants normalement destinés à une place postverbale.

Ainsi, nous sommes ici dans une même stratégie de la part du locuteur, une stratégie à la fois énonciative, discursive, informative, voire rhétorique. Plutôt qu'une construction standard, l'énonciateur choisit une structure spécialisée de focalisation (Muller, 2008) pour marquer un élément du prédicat comme ayant un rôle de focus et simultanément, pour inscrire le sujet et la partie verbale si celle-ci est explicite comme présupposés (Lambrecht, 2008). Et ces motivations communicatives entraînent des contraintes sur la structuration syntaxique de l'énoncé. On parle alors de phrases disloquées, non liées, binaires.

\subsection{Deux équivalents}

51 Les deux types d'énoncés étudiés ici suivent ainsi des schémas prosodiques identiques et présentent les mêmes rendements communicatifs. Nous pensons qu'il s'agit au demeurant de deux variantes contextuelles d'un même procédé.

Nous avons vu que pour les phrases à complément verbal extraposé, ce dernier acquérait un statut prédicatif. Il fonctionne d'ailleurs parfaitement comme prédicat d'une structure averbale dans les énoncés :

[5b] à la caisse la pesée

[24b] 1 rue Pascal Marie Agnasse son adresse

La construction verbale est affaiblie et reléguée à un niveau inférieur. La relation entre l'élément régi et le verbe est ainsi plus lâche au point que ce dernier s'apparente plus à un sujet. Il accepte alors trop peu les contrastes et semble être rajouté après coup. 
Nous pouvons donc supposer que tout locuteur, pour offrir à un membre phrastique une place de premier rang dans son énoncé, pourra employer ce type de structure avec un noyau averbal en focus. Ainsi, au lieu d'une construction liée, tout locuteur pourra préférer une structure disloquée.

Observons nos constructions et leurs équivalents canoniques :

[5a] à la caisse ils se pèsent (Sabio, 1995 : 114)

[5e] ils se pèsent à la caisse

[7a] très bien ce que tu as fait

$[7 \mathrm{~d}]$ ce que tu as fait est très bien

Nous remarquons très rapidement que les énoncés verbaux ne sont jamais structurés avec le verbe « être ». Nous notons cependant une exception, avec un sujet pronominal :

[25a] en colonie il est (Blanche-Benveniste, 1990 : 142)

Ainsi, si la structure liée correspond à une phrase avec « être », la mise en relief aboutira à une structure sans verbe avec effacement du verbe « être » :

[26a] ? La Patagonie est intéressante.

[26b] C'est intéressant, la Patagonie.

[26c] Intéressant, la Patagonie. (Blanche-Benveniste, 1990 : 141)

Lorsque la construction liée contient un verbe autre que " être ", la mise en relief avec effacement du groupe verbal est impossible :

[27a] ils nous ont piqué toute la rangée

[27b] toute la rangée ils nous ont piquée (Blanche-Benveniste, 1997 : 114)

$[27 c]$ *toute la rangée ils

puisqu'un clitique atone ne peut être formulé en construction détachée. L'exemple [27d] avec un clitique de forme forte est néanmoins possible :

[27d] *toute la rangée eux

Cependant, la prédication ne véhicule plus la même valeur. Il n'y est plus question de prédication marquant une activité comme cela l'était initialement en [27a], mais plus d'un énoncé d'ordre locatif, ou encore d'une construction incomplète.

9 Le fait que ces opérations de focalisation averbale sont possibles s'explique d'une part par le type de verbe employé ou qui sous-tend la prédication et d'autre part par le caractère toujours pronominal du sujet.

La structure avec "être » et un sujet pronominal clitique ne pourrait d'ailleurs pas aboutir, par focalisation, à une phrase averbale à deux termes :

[25b] il est en colonie

[25a] en colonie il est (Blanche-Benveniste, $1990: 142$ )

$[25 \mathrm{c}] *$ en colonie il

alors que :

[25d] ma sœur est en colonie

[25e] en colonie ma sœur

61 Nous pouvons résumer cela par le tableau suivant: 


\begin{tabular}{|c|c|c|c|}
\hline $\begin{array}{l}\text { Sujet } \\
\text { pronominal }\end{array}$ & $\begin{array}{c}\text { Verbe " être " (explicite ou } \\
\text { implicite) }\end{array}$ & Résultat & Exemple \\
\hline- & + & $\begin{array}{c}\text { Focalisation averbale (phrase } \\
\text { à deux termes) }\end{array}$ & très bien ta vie \\
\hline+ & + & $\begin{array}{c}\text { Focalisation d'un complément } \\
\text { verbal }\end{array}$ & $\begin{array}{c}\text { en colonie il } \\
\text { est }\end{array}$ \\
\hline+ & - & $\begin{array}{c}\text { Focalisation d'un complément } \\
\text { verbal }\end{array}$ & $\begin{array}{l}\text { huit cent } \\
\text { trente j'ai }\end{array}$ \\
\hline
\end{tabular}

Fig. 4 : contraintes syntaxiques des structures focalisantes à rhème extraposé.

62 Ainsi, une structure focalisante visant à mettre en avant dans la phrase un élément rhématique prendra différentes formes, selon la nature du sujet exprimé, pronominal clitique ou non, et selon le verbe qui sous-tend la construction, attributif ou non.

\section{Conclusion}

63 Nous avons cherché ici à démontrer que la structuration verbale et la structuration averbale ne sont pas deux systèmes totalement différents quand il s'agit de focaliser un argument rhématique. Ainsi, selon l'intention focalisante du locuteur, un énoncé pourra prendre différentes tournures :
[12a] À paris le vernissage ? (oral)
[12b] C'est à Paris, le vernissage ?
[12c] Le vernissage est à Paris?
$[7 \mathrm{~d}]$ ce que tu as fait est très bien
[7a] très bien ce que tu as fait (CTFP : 32)
[17b] il va m'acheter un petit mouton
[17a] un petit mouton / il va m'acheter(Blanche-Benveniste, $1990: 141$ )

La structure liée sera analysée sous un seul noyau tandis que la structure disloquée suivra un schéma différent, binaire et analysable en termes de « noyau + suffixe ». La structure disloquée sera totalement averbale si la construction liée emploie un sujet non clitique et pronominal et le verbe " être ». Le cas échéant, il s'agira d'un complément extraposé à une construction verbale. 


\section{BIBLIOGRAPHIE}

AVANZI, M. en cours. Description prosodique de constructions périphériques, paratactiques et clivées en français parlé. Thèse de doctorat. Universités de Paris Ouest Nanterre et de Neuchâtel. Accessible en ligne sur http://sites.google.com/site/mathieuavanzi/doctoral-dissertation.

Avanzi, M., MARTIN, P. 2007. L'intonème conclusif. Une fin de phrase en soi. Nouveaux cahiers de Linguistique Francaise 28 : 247-258.

BÉGUELIN, M-J. 2003. Variations entre macro- et micro-syntaxe : de quelques phénomènes de grammaticalisation. In A. SCARANO (ed.), Macro-syntaxe et pragmatique : l'analyse linguistique de l'oral. Italie / Rome : Bulzoni : 111-132.

BERRENDONNER, A. 1990. Pour une macro-syntaxe. Travaux de linguistique 21 : 25-36.

BERRENDONNER, A. 2003. Éléments pour une macro-syntaxe. Actions communicatives, types de clauses, structures périodiques. In A. SCARANo (ed.), Macro-syntaxe et pragmatique : l'analyse linguistique de l'oral. Italie / Rome : Bulzoni : 83-99.

Blanche-Benveniste, C., Bilgier, M., Rouget, C., VAn Den Eynde, K. 1990. Le Français parlé :études grammaticales. Paris : Éditions du CNRS.

BlAnCHE-BENVEniSTE, C. 1997. Approches de la langue parlée en français. L'essentiel. Paris : Ophrys. BlAnCHE-BenVENiSTE, C. 2002a. Macro-syntaxe et micro-syntaxe : les dispositifs de la rection verbale. In H.L. ANDERSEN, H. NOLKE (eds), Macro-syntaxe et macro-sémantique. Berne : Peter Lang : 95-118.

Blanche-Benveniste, C., Rouget, C., Sabio, F. 2002b. Choix de textes de français parlé, 36 extraits. Paris : Champion ${ }^{7}$.

Blasco-Dulbecco, M. 1999. Les constructions disloquées en français contemporain.Paris : Champion. Boersma, P., WeEninK, D. 2009. Praat : doing phonetics by computer (version 5.1) : http:// www.praat.org.

DelattRe, P. 1966. Les dix intonations de base du français. The French Review 40 (1) : 1-14.

DELIC 2004. Présentation du Corpus de Référence du Français Parlé. RSFP 18 : 11-42.

DeUlofeu, J. 2003. L'approche macrosyntaxique en syntaxe : un nouveau modèle de rasoir d'Occam contre les notions inutiles? Scolia 16. Strasbourg : Publications de l'Université Marc Bloch : 77-95.

DURAND, J., LAKS, B., LYCHE, Ch. 2002. La phonologie du français contemporain : usages, variétés et structure. In C. PUSCH, W. RAIBLE (eds), Romanistische Korpuslinguistik - Korpora und gesprochene Sprache / Romance Corpus Linguistics - Corpora and Spoken Language. Tübingen : Gunter Narr Verlag : 93-106.

DURAND, J., LAKS, B., LYCHE, Ch. 2005. Un corpus numérisé pour la phonologie du français. In G. W ILLIAMS (ed.), La linguistique de corpus, Actes du colloque « La linguistique de corpus », Lorient, 12-14 septembre 2002. Rennes : Presses universitaires de Rennes : 205-217. 
LACHERET-DUJOUR, A., VICTORRI, B. 2002. La période intonative comme unité d'analyse pour l'étude du français parlé : modélisation prosodique et enjeux linguistiques. Verbum 24 (1-2) : 55-73.

LAMBRECHT, K. 1994. Information structure and sentence form : Topic, focus, and the mental representation of discourse referents. Cambridge Studies in Linguistics 71. Cambridge : Cambridge University Press.

LAMBRECHT, K. 2004. Un système pour l'analyse de la structure informationnelle des phrases. L'exemple des constructions clivées. In J. FERNANDEZ-VeST, S. CARTER-THOMAS (eds), Structure informationnelle et particules énonciatives. Paris : L'Harmattan : 21-62.

LAMBRECHT, K. 2008. Contraintes cognitives sur la syntaxe de la phrase en français parlé. In D. VAN RAEMDONCK (ed.), Modèles syntaxiques. La syntaxe à l'aube du XXI e siècle. Bruxelles : Peter Lang : 247-277.

LEFEUVRE, F. 1999. La phrase averbale en français. Paris : L'Harmattan.

Lefeuvre, F. 2007. Le segment averbal comme unité syntaxique textuelle. In M. CHAROLLes, N. F oURNIER, C. Fuchs, F. Lefeuvre (eds), Parcours de la phrase. Paris : Ophrys : 143-158.

LE GofFIC, P. 1993. Grammaire de la phrase française. Paris : Hachette.

LE GOFFIC, P. 2008. Phrase, séquence, période. In D. VAN RAEMDONCK (ed.), Modèles syntaxiques. La syntaxe à l'aube du XXI siècle. Bruxelles : Peter Lang : 329-356.

MARTIN, P. 2006. Intonation du français : parole spontanée et parole lue. Estudios de Fonetica Experimental XV : 133-162.

MERTENS, P. 2006. A Predictive Approach to the Analysis of Intonation in Discourse in French. In Y. KAWAgUChI, I. Fonagy, T. MoRIgUCHI (eds), Prosody and Syntax. Amsterdam : John Benjamins : 64-101.

Morel, M-A., DANON-BoIleAu, L. 1998. Grammaire de l'intonation. L'exemple du français. Paris : Ophrys. MulLER, C. 2008. Modes d'organisation syntaxique et dépendances multiples. Micro et macrosyntaxe unifiées. In D. VAN RAEMDONCK (ed.), Modèles syntaxiques. La syntaxe à l'aube du XXI siècle. Bruxelles : Peter Lang :213-229.

PERROT, J. 1978. Fonctions syntaxiques, énonciation, information. In Bulletin de la Société Linguistique de Paris LXXIII (1) : 85-101.

PERROT, J. 1994. L'ordre des mots dans l'énoncé-message : principes et illustrations. Tema 1 : 33-50.

PerRot, J. 1995. Éléments pour une typologie des structures informatives. In Mémoires de la Société Linguistique de Paris. Tome II : La phrase : énonciation et information. Louvain : Peeters.13-26.

Riegel, M., Pellat, J-C., Rioul, R. 1994. Grammaire méthodique du français. Paris : Presses universitaires de France.

SABIO, F. 1995. Micro-syntaxe et macro-syntaxe : l'exemple des « compléments antéposés » en français. Recherches sur le français parlé 13 : 111-155.

SABIO, F. 2006a. Phrases et constructions verbales : quelques remarques sur les unités syntaxiques dans le français parlé. Constructions verbales et production de sens, Actes du colloque organisé à Besançon les 26, 27 et 28 janvier 2006. Besançon : Presses universitaires de Franche-Comté : 127-140.

SABIO, F. 2006b. L'antéposition des complémentsdans le français contemporain : l'exemple des objets directs. Lingvistica Investigationes 29 : 173-182. 
SABIO, F. 2007. La description de « l'ordre des mots » confrontée à la diversité des usages : quelques observations sur la place des compléments en français. Cahiers de l'Association for French Language Studies 13 (1) : 18-32.

\section{NOTES}

1. Les exemples indexés "CTFP : numéro du texte " sont issus du corpus publié et accessible de Blanche-Benveniste et al. (2002b) : Choix de Textes de Français Parlé.

2. L'énoncé [3] est composé de trois périodes intonatives, au sens de Lacheret et Victorri (2002). De telles unités prosodiques sont délimitées ici par les doubles barres obliques //.

3. Les exemples indexés «CRFP, DELIC » sont issus du Corpus de Référence du Français Parlé, constitué par l'équipe DELIC et rassemblant 134 enregistrements.

4. Dans la terminologie aixoise, les éléments postfixe et suffixe s'opposent au niveau prosodique. Le postfixe, énoncé en position post-finale, est exprimé sans aucun contour mélodique (contour plat: intonation basse ou sans intonation) et de ce fait, sans syllabe accentuée. À l'inverse, une structure «noyau-suffixe » comprend deux éléments aux contours de modalités indépendants (Avanzi et Martin, 2007 ; Martin, 2006).

5. Les exemples indexés "PFC» sont issus du corpus Phonologie du Français Contemporain (Durand et al., 2002 et 2005) : http://www.projet-pfc.net.

6. Voir à ce sujet l'intonation d'implication de Delattre (1966).

7. Les exemples extraits de ce corpus sont suivis de la mention «CTFP» assortie du numéro du texte dont ils sont issus.

\section{RÉSUMÉS}

Cet article se propose de rapprocher deux types de structures couramment employées en français parlé et qui présentent toutes deux la particularité d'être énoncées en deux temps. L'une est cependant construite autour d'un verbe, tandis que la seconde est structurée à partir d'un constituant averbal :

[a] à la caisse ils se pèsent (Sabio, $1995: 114)$

[b] très bien ta vie (CTFP : 32)

Nous montrerons que ces deux types d'exemples suivent tout d'abord un même schéma prosodique. Les constructions [a] et [b] sont prononcées en deux groupes intonatifs. Le premier est porteur d'une intonation modale qui le rend autonome tandis que le second est prononcé comme un appendice. Ensuite, nos deux réalisations présentent les mêmes rendements communicatifs. Elles résultent d'une même opération de focalisation, opération visant à placer en tête de phrase un argument à vocation rhématique. Nous proposerons alors une même interprétation de ces constructions à analyser en termes de "noyau - suffixe ». Enfin, nous verrons que ces deux exemples peuvent être analysés comme des variantes contextuelles d'un même procédé contraintes par le type de verbe et la nature du sujet qui sous-tendent la construction.

The aim of this paper is to compare two types of (syntactic) structures which are frequently used in spoken French. While they are both uttered in two parts, one structure contains a verb and the 
other is based on a verbless element:

[a] à la caisse ils se pèsent [Sabio, $1995: 114]$ = at the checkout, they are weighed

[b] très bien ta vie [CTFP : 32] = very pleasant, your life

We will show that such examples follow the same prosodic pattern. Construction $[\mathrm{a}]$ and $[\mathrm{b}]$ are uttered with a pause which splits them into two intonation groups. The first conveys a modal intonation which makes it autonomous while the second sounds like an appendix or extension. Furthermore, the same pragmatic effect is obtained by both structures, as they result from the same focalisation process, entailing the fronting of a rhematic element.

We will hence suggest an identical interpretation for such structures, as they can be analysed as a 'nucleus' + a 'suffix'. We will finally show that these two examples can be analysed as contextual variants for the same process, and that these variants are the result of a constraint created both by the type of verb and by the syntactic type of the subject which underlie the construction.

\section{INDEX}

Keywords : focusing, oral, predicate, sentence, syntax, verbless

Mots-clés : averbal, focalisation, oral, phrase, prédicat, syntaxe

\section{AUTEUR}

\section{NOALIG TANGUY}

Université Paris 3 Sorbonne Nouvelle - Lattice UMR 8094noaligtanguy@gmail.com 